\title{
RIN-Penalty Mitigation and Transmission Performance Improvement Using Forward Propagated Broadband First Order Raman Pump
}

\author{
Mingming Tan, Md Asif Iqbal, Lukasz Krzczanowicz, Ian. D. Phillips, Atalla El-Taher, \\ Wladek Forysiak, and Paul Harper \\ 1 Aston Institute of Photonic Technologies, \\ Aston University, \\ Birmingham, B4 7ET, UK \\ m.tan1@aston.ac.uk
}

\begin{abstract}
We demonstrate that using a broadband pump enables forward-propagated first order distributed Raman amplification by mitigating RIN-associated penalty. This extends the reach of $10 \times 120 \mathrm{~Gb} / \mathrm{s}$ DP-QPSK WDM transmission up to $7499 \mathrm{~km}$, compared with other commercially available pumps. Moreover, using this Raman scheme maintains uniform/symmetric signal power distribution and requires low pump power.
\end{abstract}

Keywords - coherent communications; Raman amplification;

\section{INTRODUCTION}

As coherent transmission systems move to higher order modulation formats a higher signal-to-noise ratio (SNR) is required. Using distributed Raman amplification (DRA) can effectively reduce amplified spontaneous emission (ASE) noise and improve the signal-to-noise ratio, in comparison with discrete amplification. In DRA, using bidirectional pumping can distribute the signal power uniformly along the fibre and provide superior noise performance over backward-propagated (BW) pumping only [1]. However, a major difficulty of using forward Raman pump is relative intensity noise (RIN) and its associated penalty, in particular for long-haul repeatered transmission systems $[2,3]$. We previously showed a Raman amplification based on random distributed feedback (DFB) fibre laser with bidirectional second-order pumping improved the maximum reach by $0.7 \mathrm{~dB}$, compared with $\mathrm{BW}$-pumping only. This indicates the RIN penalty had been significantly mitigated [4]. However, the main drawback of this scheme was due to the use of second order pump in the forward direction with no seed of first order pump, the Raman gain efficiency near the span input was very low. In order to improve the Raman gain efficiency, using first order FW-pump is the simplest way.

Here, we present a detailed evaluation of the long-haul transmission performance in a $100 \mathrm{G}$ DP-QPSK WDM coherent transmission system, by using different forwardpropagated first order pumps with different RIN/power levels based on Raman fibre laser based amplification. We demonstrate that using a broadband Raman fibre laser with relatively low RIN level can extend the maximum transmission distance without increasing the RIN of the signal, and we compare it with other commercially available pump lasers, i.e. semiconductor laser diodes and narrowband Raman fibre laser. Using the proposed scheme gives a maximum reach of 7499 $\mathrm{km}$, compared with $4999 \mathrm{~km}$ using a semiconductor laser diode and $1500 \mathrm{~km}$ using a narrowband Raman fibre laser. Furthermore, unlike other pump lasers, the RIN of the output signal using this broadband pump doesn't increase dramatically when the pump power increases. Therefore, different signal power profiles along the fibre can be achieved in order to satisfy the span requirement for different nonlinearity compensation techniques.

\section{EXPERIMENTAL SETUP OF RAMAN AMPLIFIED TRANSMISSION} SYSTEMS

To quantify the impact on the long-haul transmission performance using different $\mathrm{FW}$-propagated pump lasers, a recirculating loop experiment was conducted using the set-up shown in Fig. 1. Ten DFB lasers spaced at $100 \mathrm{GHz}$ (from $1542.94 \mathrm{~nm}$ to $1550.12 \mathrm{~nm}$ ) were combined with a $100 \mathrm{kHz}$ linewidth tuneable laser ("channel under test"). The combined signals were QPSK modulated at 30G Baud. A PM EDFA was used to amplify the signal. The $10 \times 120 \mathrm{~Gb} / \mathrm{s}$ DP-QPSK signals were generated through a polarisation multiplexer before the input of the recirculating loop. The transmission fibre in the recirculating loop was $83.32 \mathrm{~km}$ standard SMF-28 fibre with a total loss of $\sim 17.6 \mathrm{~dB}$, including $\sim 16.5 \mathrm{~dB}$ from the fibre and $\sim 1.1 \mathrm{~dB}$ from pump signal combiners. A gain flattening filter (GFF) was used to flatten the spectrum after the Raman link. The $\sim 12 \mathrm{~dB}$ loss from GFF, 50/50 coupler, two acousto-optic modulators (AOM), and WDM couplers, was compensated using a single stage EDFA at the end of the loop. The output signal was de-multiplexed by a tuneable filter and amplified by an EDFA. The receiver was a standard polarisation-diverse coherent detection set-up, and the signals were captured with four photo-detectors using an $80 \mathrm{GSa} / \mathrm{s}, 36 \mathrm{GHz}$ bandwidth oscilloscope. DSP was used with standard algorithms for signal recovery and transmission impairments compensation. Q factors were calculated from bit-wise error counting, and averaged over two million bits.

Schematic diagrams for the Raman configurations tested are shown in Fig. 2. For all configurations the on-off gain was set to compensate the $16.5 \mathrm{~dB}$ loss of the fibre. As a baseline, 
second order backward only pumping at $1366 \mathrm{~nm}$ with the FBG near the output end of the span was used. The FBG was centred at $1452 / 1455 \mathrm{~nm}(0.5 \mathrm{~nm} 3 \mathrm{~dB}$ bandwidth and $95 \%$ reflectivity). The difference in centre wavelength was to match the wavelength of the FW-pump. The first-order random fibre laser at $1455 \mathrm{~nm}$ was generated due to the resonant mode reaching the lasing threshold in a distributed cavity formed by Rayleigh scattering and an FBG [4]. To enhance the signal-tonoise ratio, three types of $\mathrm{FW}$-propagated pumps were used, as forward pumping at $1455 \mathrm{~nm}$ could amplify the signal near the input section of the fibre and consequently reduce the signal power variation (SPV). The first type of forward pump was a commercially available broadband $(\sim 8 \mathrm{~nm} 3 \mathrm{~dB}$ bandwidth) Raman fibre laser with the RIN level of $-138 \mathrm{~dB} / \mathrm{Hz}$ and $5 \mathrm{~W}$ maximum output power. In comparison, semiconductor laser diodes with $0.8 \mathrm{~nm} 3 \mathrm{~dB}$ bandwidth and $-135 \mathrm{~dB} / \mathrm{Hz}$ RIN level were used as the second forward pump configuration. The pump output was depolarised by combining two laser diodes through a polarisation beam combiner, and the output power was up to $\sim 400 \mathrm{~mW}$. A narrowband $(0.5 \mathrm{~nm} 3 \mathrm{~dB}$ bandwidth) Raman fibre laser with maximum output power of $5 \mathrm{~W}$ and considerably high RIN $(-113 \mathrm{~dB} / \mathrm{Hz})$ was used as the third forward pump configuration.

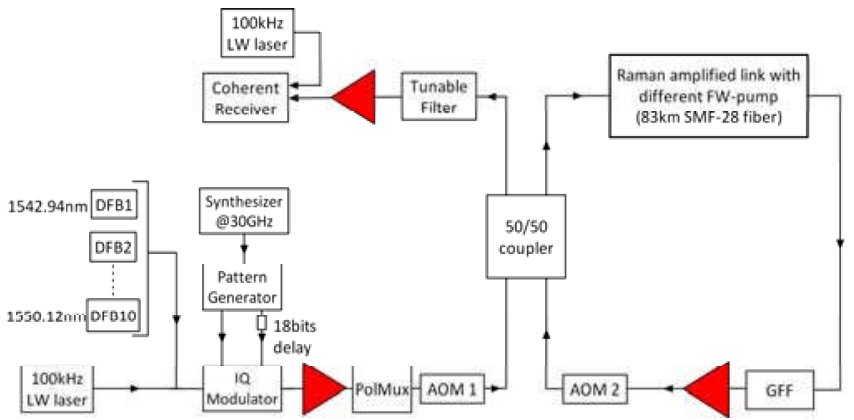

Figure 1. Experimental setup of long-haul transmission system

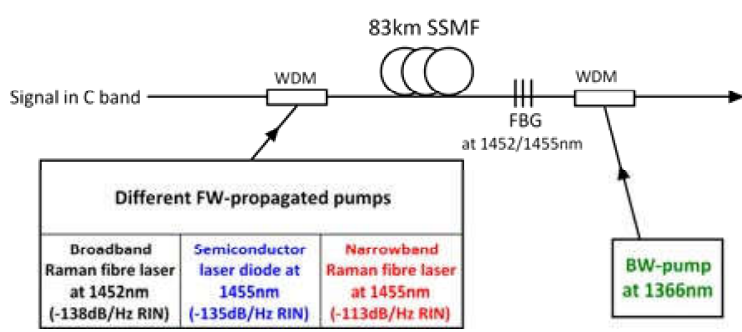

Figure 2. Schematic diagrams of Raman amplification schemes using different types of forward pumps

Signal power profiles along the transmission span measured using a modified optical time-domain reflectometer (OTDR) are shown in Fig. 3. Using BW-pumping only resulted in an SPV of $\sim 6 \mathrm{~dB}$. In a bi-directionally pumped setup, signal power variation was reduced to $4 \mathrm{~dB}$ using $89 \mathrm{~mW}$ FW-pump power $(100 \mathrm{~mW}$ for the broadband $\mathrm{FW}$-pump because of larger $3 \mathrm{~dB}$ bandwidth), and only $\sim 2.5 \mathrm{~dB}$ using $166 \mathrm{~mW}$ FW-pump power (186 $\mathrm{mW}$ for the broadband pump). Assuming no RINassociated penalty from the forward pump is introduced, this reduction in signal power variation should lead to the improvement of the transmission performance. Here, note that signal power profiles are mainly related to pump power regardless of forward pump type, so the signal power profiles using different forward pumps are the same.

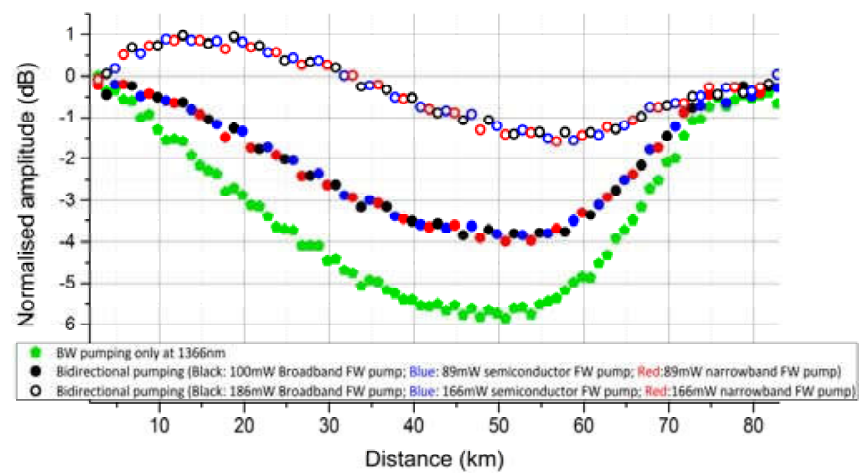

Figure 3. Measured signal power profiles along the fibre using BW-pump only, $89-100$, and $166-186 \mathrm{~mW}$ FW pump power (signal power profiles are mainly related to pump power regardless of forward pump type)

The RIN of the signal could lead to significant penalty on the long-haul transmission performance [3]. RIN of the signal at the span output were experimentally investigated for all the pumping schemes and are shown in Fig. 4. The detailed setup for RIN measurement can be found in [3]. In Fig. 4, using 100 $\mathrm{mW}$ broadband Raman fibre laser, the RIN of the output signal was similar to BW-pumping only over the whole frequency range, which indicates RIN-induced penalty didn't exist on the transmission [4]. Increasing the pump power to $186 \mathrm{~mW}$, the RIN was increased slightly by $2 \mathrm{~dB}$ at low frequency range below $20 \mathrm{MHz}$. In comparison, the RIN dramatically increased by $10 \mathrm{~dB}$ at low frequency range using semiconductor laser with $89 \mathrm{~mW}$ pump power, regardless of similar pump RIN to the broadband pump. Using high RIN narrowband pump made the RIN worse, up to $20 \mathrm{~dB}$ increase in the signal RIN, which may result in critical penalty on the transmission performance.

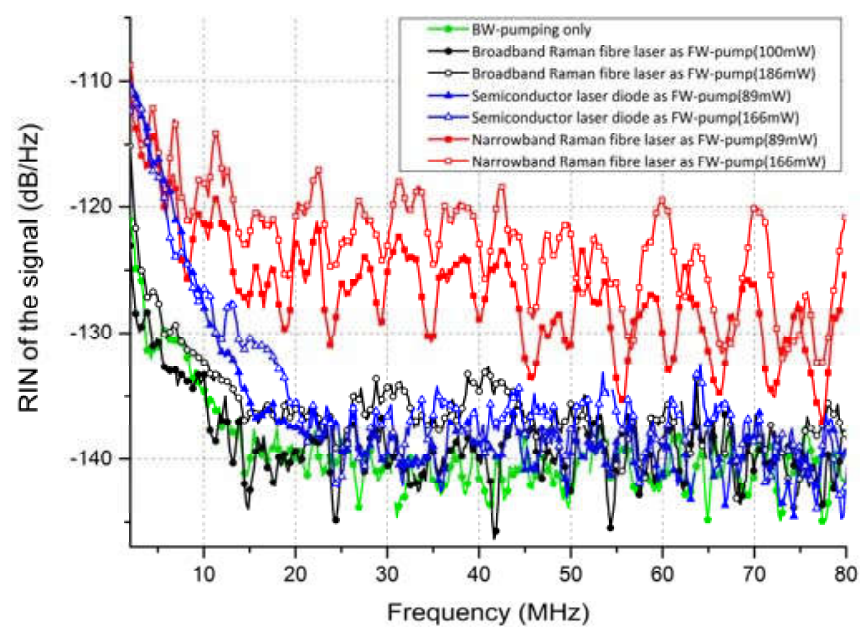

Figure 4. RIN of the output signal using different forward pumps

\section{TRANSMISSION RESULTS AND DISCUSSIONS}

Fig. 5 shows Q factors versus signal launch power per channel at $3333 \mathrm{~km}$ for different Raman configurations except that narrowband Raman fibre laser was used as the FW-pump: that configuration could not achieve $3333 \mathrm{~km}$, so results are shown at $1500 \mathrm{~km}$ which was the maximum distance that could 
be achieved. Fig. 6 shows $Q$ factors versus transmission distances. The circled points in Fig. 6 are the $\mathrm{Q}$ factors at optimum launch powers shown in Fig. 5.

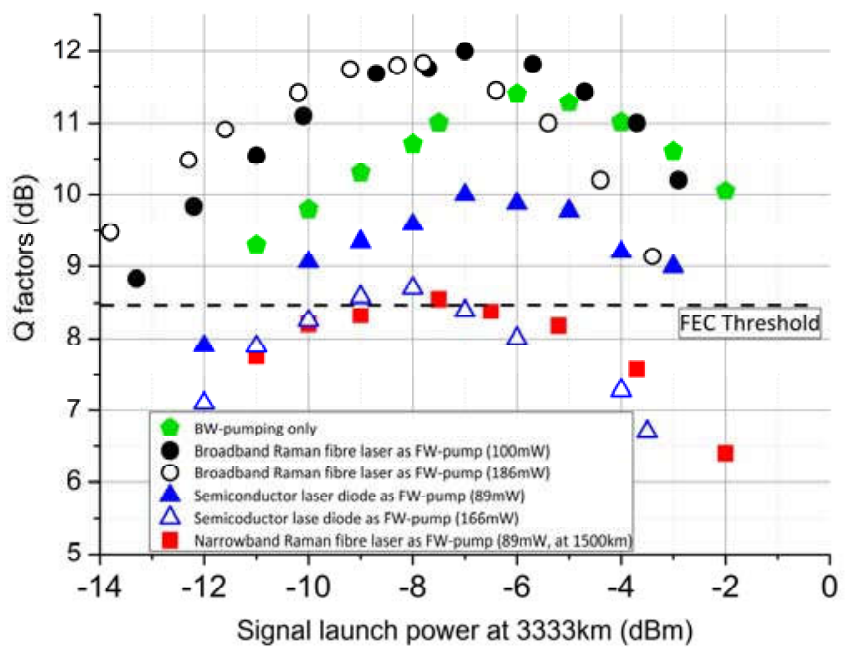

Figure 5. Q factors versus signal launch power per channel

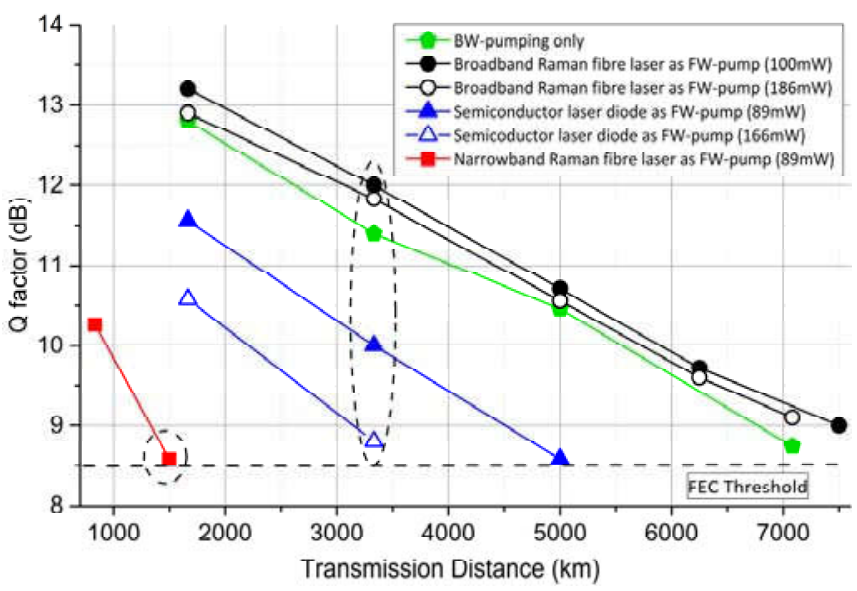

Figure 6. Q factors versus transmission distances

Using the BW-pumping only, the best $\mathrm{Q}$ factor was $11.4 \mathrm{~dB}$ at $-6 \mathrm{dBm}$ optimum signal launch power. Consequently, BWpumping only scheme gave a transmission distance of 7082 $\mathrm{km}$. Using broadband Raman fibre laser as the FW-pump, the optimum Q factor was improved to $12 \mathrm{~dB}$ with $100 \mathrm{~mW}$ FWpump power, and the maximum reach was extended to 7499 $\mathrm{km}$. This was because the signal RIN stayed the same and therefore the Q factor was improved due to the amplifier noise figure reduction and uniform signal power distribution $[3,4]$. As expected from Fig. 3, the impact of nonlinearity reduced the optimum launch power to $-7 \mathrm{dBm}$, as $\mathrm{FW}$-pumping resulted in a higher average signal power. With $166 \mathrm{~mW}$ forward pump power, a Q factor of $11.83 \mathrm{~dB}$ was achieved at the optimum launch power of $-8 \mathrm{dBm}$. The maximum reach was slightly decreased to $7082 \mathrm{~km}$. This was due to the slight increase in the signal RIN. In the FW-propagated semiconductor laser diode setup, the optimum Q factor at $3333 \mathrm{~km}$ was only $10 \mathrm{~dB}$ with $89 \mathrm{~mW}$ and $8.7 \mathrm{~dB}$ at $166 \mathrm{~mW}$. The maximum reach was $4999 \mathrm{~km}$, much worse in comparison with BW-pumping only and FW-propagated broadband Raman pump. When using narrowband Raman fibre laser as the forward pump, the transmission performance was much worse because of its relatively high RIN $(-113 \mathrm{~dB} / \mathrm{Hz})$ and narrow $3 \mathrm{~dB}$ bandwidth, limiting the maximum reach to only $1500 \mathrm{~km}$. Here, we noted that using higher FW-pump power degraded the performance even with very low launch power per channel (i.e. $-10 \mathrm{dBm}$ ), as the impact of fibre nonlinearity was negligible. Q factors and received spectra at maximum transmission distances for all the Raman configurations are available at the conference. All the channels were above FEC threshold $\left(3.8 \times 10^{-3}\right.$ in bit error rate).

Therefore, to improve the transmission performance using first order FW-propagated pump in DRA for long-haul transmission, the required pump should essentially have the low RIN level (i.e. $<-135 \mathrm{~dB} / \mathrm{Hz}$ ). Low stimulated Brillion scattering (SBS) is also required, but this is easily achieved due to the low pump power being used [6]. More importantly, a broadband $3 \mathrm{~dB}$ bandwidth (i.e. $8 \mathrm{~nm}$ ) is crucial to reduce the RIN transfer.

\section{CONCLUSION}

We have demonstrated that using a broadband low RIN FWpropagated pump in distributed Raman amplification can effectively extend the reach of $10 \times 120 \mathrm{~Gb} / \mathrm{s}$ DP-QPSK longhaul transmission without increasing the RIN of the signal, in comparison with other commercially available Raman pump lasers. This scheme offers the best transmission performance, maintains only $2.5 \mathrm{~dB}$ signal power variation, and requires low pump power. In addition, using such a scheme can provide a symmetric link which maximises the benefit of nonlinearity compensation using mid-link OPC, or a low signal power variation for a nonlinear Fourier transform based transceiver.

\section{ACKNOWLEDGMENT}

This work was funded by UK EPSRC Programme Grant UNLOC EP/J017582/1, FP7 ITN programme ICONE (No. 608099), and industrial support from II-IV. We thank C. Wang, Z. Sun, and L. Zhang for providing FBGs.

\section{REFERENCES}

[1] J. D. Ania-Castañón, "Quasi-lossless transmission using second-order Raman amplification and fibre Bragg gratings," Opt. Express, vol. 12, no. 19, pp. 4372-4377, 2004.

[2] L. Xu et al., "Experimental verification of relative phase noise in Raman amplified coherent optical communication system," Journal of Lightwave Technology, vol.34, no. 16, pp. 3711-3716, 2016.

[3] M. Tan et al., "Evaluation of $100 \mathrm{G}$ DP-QPSK long-haul transmission performance using second order co-pumped Raman laser based amplification," Opt. Express, vol. 23, pp. 22181-22189, 2015.

[4] M. Tan et al., "Transmission performance improvement using random DFB laser based Raman amplification and bidirectional second-order pumping," Opt. Express, vol. 24, pp. 2215-2221, 2016.

[5] M. Tan et al., "RIN Mitigation in Second-Order Pumped Raman fibre laser based amplification," in ACP 2015, paper AM2E.6.

[6] Y Y. Ohki et al., "Pump laser module for co-propagating Raman amplifier," Fukukawa Review, vol. 24, no. 1pp. 6-12, 2003 